\title{
Fixed-Time Convergent Guidance Law with Impact Angle Control
}

\author{
Zhongtao Cheng $\mathbb{D},{ }^{1}$ Hao Wu, ${ }^{2}$ Bo Wang $\mathbb{D},{ }^{1}$ Lei Liu $\mathbb{D},{ }^{1}$ and Yongji Wang $\mathbb{D}^{1}$ \\ ${ }^{1}$ National Key Laboratory of Science and Technology on Multispectral Information Processing, \\ School of Artificial Intelligence and Automation, Huazhong University of Science and Technology, Wuhan, China \\ ${ }^{2}$ Beijing Aerospace Automatic Control Institute, Beijing, China \\ Correspondence should be addressed to Bo Wang; wb8517@hust.edu.cn
}

Received 4 April 2020; Accepted 12 May 2020; Published 29 May 2020

Academic Editor: Jianquan Lu

Copyright (C) 2020 Zhongtao Cheng et al. This is an open access article distributed under the Creative Commons Attribution License, which permits unrestricted use, distribution, and reproduction in any medium, provided the original work is properly cited.

\begin{abstract}
The existing convergence control guidance laws are designed via the Lyapunov asymptotic stability theory or finite-time stability theory. However, guidance law based on the Lyapunov asymptotic stability theory would lead the states to zero only as time approaches infinity, which is imperfect theory. The convergence time for guidance laws based on finite-time stable theory is dependent on the initial states. A fixed-time convergent guidance law with impact angle control is proposed in this paper. The proposed guidance law consists of two parts. One is the heading error angle shaping term, and the other is the bias term to achieve the desired impact angle. The guidance command is continuous during the engagement without utilizing the switching logics. Unlike the existing guidance law in the literature, the fixed-time stability theory is utilized to ensure the impact angle error to converge to zero before the interception. Furthermore, the convergence rate is merely related to control parameters. Simulations are carried out to illustrate the effectiveness of the proposed guidance law.
\end{abstract}

\section{Introduction}

In the design of the missile guidance system, the primary objective is to reduce the relative range and achieve zero miss-distance attacks. In modern warfare, the mission is more diverse, and the battlefield is more complex $[1,2]$. For example, the missile needs to avoid the defensive system by using a detour in certain missions. Also, specific impact angles to the weakest part of the target are effective for anti-tank or anti-ship missiles. Primitive guidance laws that only achieve the primary objective cannot accommodate to modern war. Hence, studies on the impact angle control guidance (IACG) have very high strategic significance [3-5].

Since the first study on impact angle control on a reentry vehicle [6], various guidance and control schemes are applied in the design of IACG, such as the proportional navigation (PN) law and its variants, optimal control theory, nonlinear control theory, and other geometry methods.

Due to the simple structure and optimality, many studies on IACG are based on PN and its variants. Apart from the terminal position constraint, specific impact angles with high precision are achieved in simple proportional forms with nonlinear adaptive parameters [7]. A two-stage PN impact angle control law was present in [8], the orientation navigation stage leads the missile to certain switching states, which depend on the initial states of the missile and the desired impact angle, and the final stage leads the missile to the target with the desired impact angle. As an extension of the work in [8], both the heading error constraint and the impact angle constraint were considered in the two-stage PN [9]. Switching logic was used in two-stage PN methods; however, this would lead to an accumulated impact angle error because of autopilot dynamics in practice.

After the optimal control theory was utilized in the impact angle constraint research in [6], some other guidance laws in the literature also solved the impact angle control problem via this theory. In [10], considering different missile dynamics, a generalized optimal guidance law was offered in the state feedback form, and its characteristics were also investigated. After obtaining a new time-to-go estimation method, a novel optimal 
impact angle control guidance law was proposed for constant velocity missile [11]. A new linear optimal IACG was proposed in [12]; unlike the traditional framework, the linearization was not conducted around the initial LOS angle but around a nominal circular trajectory.

The nonlinear control theories, such as the Lyapunov stability theory and the sliding mode theory, were also adopted in the design of IACG. In [13], to hit the target with a specific angle, the Lyapunov candidate function was augmented with an impact angle error term. A recent Lyapunov stability theory-based guidance law was found in [14], the candidate function was proposed to reduce the heading error angle, and a two-stage IACG was proposed for hitting the target in all aspects. However, the states would converge to zero only as time approached infinitely for the Lyapunov-based guidance law, theoretically. Hence, some other studies involved the finite-time convergence IACG. In [15], an IACG law that insured the convergence of the line of sight (LOS) angle in finite-time was proposed from the sliding mode control theory. In [16], a finite-time convergent IACG based on the nonsingular terminal sliding mode control (NTSMT) method was proposed, and the resulting guidance law can hit the target with a desired impact angle. Another finite-time convergent guidance law was found in [17]. However, a guidance law based on the Lyapunov asymptotic stability theory would lead the states to zero only as time approaches infinity, which is imperfect theory. Also, the convergence time for guidance laws based on finite-time stable theory is dependent on the initial states.

In addition to the methods described above, the geometric and polynomial approach has also been adopted to derive the IACG law. The first appearance of the polynomial guidance was found in [18]. After that, an augmented impact angle control polynomial guidance law considering acceleration constraint was proposed in [19]. The guidance command was present in the form with unknown coefficients corresponding to the terminal constraints. To control impact time and angle, the guidance command was proposed as a function of range-to-go in [20]. A very recent research involved the geometric and polynomial approach found in [21], and the resulting guidance law was proposed in the adaptive form.

In this study, to overcome the limitations of the existing studies, a nonswitching fixed-time convergent guidance law with impact angle control is proposed. First, a heading error-shaping method is introduced to ensure the successful impact of the target. Also, the terminal characteristics of this error-shaping method are summarized. Then, the fixed-time stability theory is applied to design a bias term, which can ensure the fixed-time convergence of the impact angle error.

The rest of this paper is organized as follows. Preliminary on fixed-time stability is introduced in Section 2. In Section 3, the heading error shaping and the design of the bias term are offered. In Section 4, the effectiveness of the proposed strategy is verified through different numerical simulations. Finally, the conclusion can be found in Section 5.

\section{Fixed-Time Stability Theory of a Nonlinear System}

Before deriving the guidance law, it is obliged to introduce some basic concepts of fixed-time stability theory [22].

Definition. The following nonlinear system is considered:

$$
\begin{aligned}
\dot{x}(t) & =f(t, x(t)), \\
x(0) & =x_{0},
\end{aligned}
$$

where the state and the upper semicontinuous mapping are denoted by $x(t) \in R^{l}$ and $f: R^{+} \times R^{n} \longrightarrow R^{n}$, respectively. The state is fixed-time stability if it is globally finite-time stable; meanwhile, the function of the settling time $T\left(x_{0}\right)$ is restricted by a real positive number $T_{\text {max }}$, i.e., $T\left(x_{0}\right) \leq T_{\max }, \forall x_{0} \in R^{l}$. The definition can be stated mathematically as

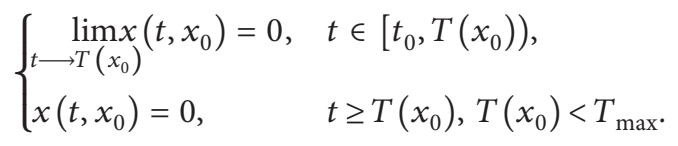

It should be noted that the settling time in (2) is independent of the initial states. Denote by $D^{*} \varphi(t)$ the upper right-hand derivative of a function $\varphi(t)$, $D^{*} \varphi(t)=\lim _{h \rightarrow+0}(\varphi(t+h)-\varphi(t)) / h$. Also, the fixed-time stability under the Lyapunov criterion is presented in Lemma 1.

Lemma 1. Suppose a continuous positive definite and radially unbounded function as $V(x): R^{n} \longrightarrow R^{+} \cup\{0\}$, such that

$$
D^{*} V(x(t)) \leq-m V^{p}(x(t))-n V^{q}(x(t)),
$$

for $m, n>0, p=1-(1 / 2 \gamma), q=1+(1 / 2 \gamma), \gamma>1$. Then, the origin is fixed-time stable for the system in (1), and the settling time is given by

$$
T\left(x_{0}\right) \leq T_{\max }:=\frac{\pi \gamma}{\sqrt{m n}}
$$

Remark 1. Different from finite-time stability theory, the bound of the settling time function for fixed-time stability theory is merely connected with the design parameters.

\section{Problem Statement and Guidance Law Design}

In this section, the two-dimensional kinematic equations that stand for the engagement geometry between the target and the missile are formulated, and the objective of the impact angle control problem is elaborated. Then, the concept of fixed-time convergence of the impact angle error is introduced, and the impact control guidance law is designed.

3.1. Engagement Geometry and Problem Statement. The missile-to-target system is denoted in Figure 1, where the planar engagement geometry is considered. In Figure 1, $r$ denotes the relative range between the missile and target. $V$ 


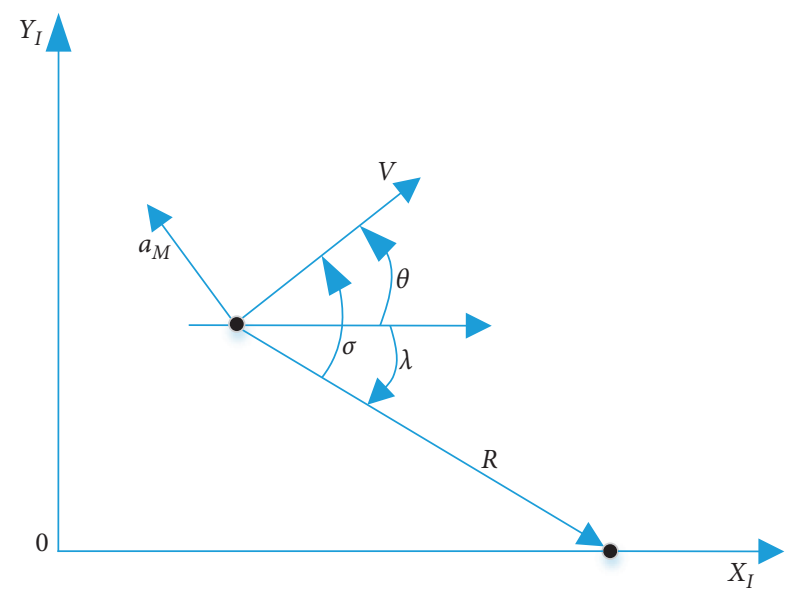

FIGURE 1: Engagement geometry.

and $a$ represent the missile's speed and later acceleration, respectively. $\theta, \sigma$, and $\lambda$ stand for the flight path angle, the heading error angle, and the line of the LOS angle, respectively.

Assume the counterclockwise direction as the positive direction for the aforementioned angles. Under the small angle of attack assumption, the geometry relationship between the angles can be expressed as

$$
\sigma=\theta-\lambda
$$

The kinematic equations for the missile to intercept the target can be obtained as

$$
\begin{aligned}
& \dot{r}=-V \cos \sigma, \\
& \dot{\lambda}=\frac{-V \sin \sigma}{r}, \\
& \dot{\theta}=\frac{a_{M}}{V} .
\end{aligned}
$$

The basic requirement for a guidance law is to lead to missile to hit the target, which is also the primary objective for the guidance law design in this paper. Apart from the primary objective, the additional objective is that the impact angle should converge to the desired value as the engagement proceeds, which can be expressed mathematically as

$$
\theta \longrightarrow \theta_{d} \text {, as } t \longrightarrow t_{s} \leq t_{f} \text {. }
$$

It should be noted that $\theta_{d}$ in (9) refers to the desired value for the impact angle, and $t_{s}$ should be smaller than the final impact time.

3.2. Primary Guidance Law with Zero Miss-Distance. In this subsection, the primary objective of the guidance law is achieved through the heading error angle shaping method, and the terminal characteristics of this method are briefly summarized.

Since the angle of attack is assumed to be small in the previous subsection, the missile will hit the target with zero miss-distance by zeroing the heading error. Hence, the zero- miss distance design problem is transformed into the heading error angle control problem. In this regard, the Lyapunov candidate function is proposed as

$$
W_{1}=\sin ^{2} \frac{\sigma}{2} .
$$

The time derivative of (10) is

$$
\dot{W}_{1}=\frac{\sin \sigma \cdot \dot{\sigma}}{2} .
$$

To meet the asymptotic stability requirement, the candidate function should be positive definite $W_{1}$, and its derivative $\dot{W}_{1}$ should be negative definite.

Theorem 1. The asymptotic stability condition can be met if the heading error satisfies

$$
\dot{\sigma}=\frac{c V}{r} \sin \sigma, \quad c \geq 1,
$$

where $c$ controls the speed of the heading error rate.

Proof. Combining equations (11) and (12) yields

$$
\dot{W}_{1}=-\frac{c V}{2 r} \sin ^{2} \sigma .
$$

It can be concluded from (10) that $W$ is positive definite, and (13) denotes that $\dot{W}_{1}$ is negative definite. Hence, the Lyapunov asymptotic stability condition is satisfied, and the proof of Theorem 1 is completed.

The Lyapunov asymptotic stability theory-based method only ensures convergence when the time approaches infinity. Obviously, this is imperfect theory. Hence, before prolonging the method to achieve the additional impact angle objective, the terminal characteristics of this guidance strategy are briefly summarized.

Dividing (12) by (6) yields

$$
\frac{\mathrm{d} \sigma}{\tan \sigma}=\frac{c}{r} \mathrm{~d} r .
$$

By solving a separable differential (15), $\sigma$ can be expressed as a function of $r$ as

$$
\sin \sigma=\left(\frac{r}{r_{0}}\right)^{c} \sin \sigma_{0} .
$$

It can be concluded from (15) that $\sin \sigma \longrightarrow 0$ as $r \longrightarrow 0$, if $c \geq 1$. As a result, the heading error can converge to zero at the instant of attack.

The derivative of (5) to time is

$$
\dot{\sigma}=\dot{\theta}-\dot{\lambda} .
$$

Substituting (7) and (12) into (16) yields

$$
\dot{\theta}=-\frac{(c+1)}{c} \dot{\sigma} .
$$

In addition, combining (12) and (15) yields 


$$
\dot{\sigma}=-\frac{c V}{r_{0}^{c}}(r)^{c-1} \sin \sigma_{0} .
$$

Hence, the guidance command that leads the missile to the target with zero miss-distance can be acquired as

$$
a^{L}=-\frac{(c+1) V^{2}}{r_{0}^{c}}(r)^{c-1} \sin \sigma_{0},
$$

where the superscript $L$ stands for the terminal states of the missile under the heading error-shaping method. By combining (7) and (12), we have

$$
\dot{\lambda}=\frac{\dot{\sigma}}{c} \text {. }
$$

Integrating both sides of (20) from current time $t$ to final time $t_{f}$ yields

$$
\lambda_{f}^{L}=\lambda+\frac{\sigma_{f}^{L}-\sigma}{c} .
$$

Substituting (5) into (21) yields

$$
\theta_{f}^{L}=\theta-\frac{c+1}{c} \sigma
$$

Remark 2. The heading error-shaping method can ensure the convergence of the guidance command at the instance of interception, and the terminal intercept angle under this method can be calculated from equation (22). Also, the impact angle error can be acquired.

3.3. Impact Angle Control Guidance Law Design. In this section, the heading error-shaping method is further investigated. In addition, the impact angle control objective is achieved. The difference between $\theta_{d}$ and $\theta_{f}^{L}$ calculated from (22) is regarded as the impact angle error, which can be expressed as follows:

$$
e_{\theta f}=\theta_{f}^{L}-\theta_{d}=\theta-\frac{c+1}{c} \sigma-\theta_{d}
$$

The time derivative of $(23)$ is

$$
\dot{e}_{\theta f}=\dot{\theta}-\frac{c+1}{c} \dot{\sigma}
$$

If the impact angle error can converge to zero before the final time, the additional objective expressed in (9) can be achieved. Substituting (8) and (19) into (24) yields

$$
\dot{e}_{\theta f}=\frac{a_{M}}{V}+\frac{(c+1) V \sin \sigma}{r}=\frac{a_{M}-a^{L}}{V}=\frac{u}{V},
$$

where $u$ is defined as the bias term of the guidance command, and the purpose of introducing the bias term $u$ is illustrated in Figure 2, which aims at governing the dynamics of the impact angle error to zero. Hence, the objective of impact angle control will be achieved through the design of $u$. The impact angle control guidance command is constructed as

$$
a_{M}=a^{L}+u
$$

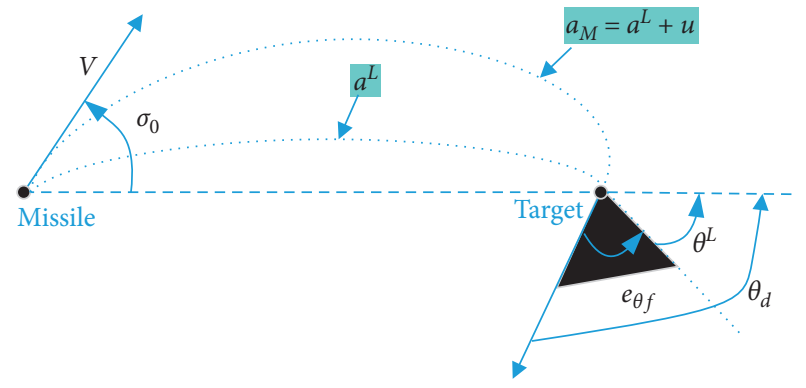

Figure 2: The purpose of introducing the bias term $u$.

Theorem 2. If the bias term $u$ can make the impact angle error of the missile satisfying

$$
e_{\theta f}\left[\dot{e}_{\theta f}+\frac{m}{2} e_{\theta f}^{1-(1 / \gamma)}+\frac{n}{2} e_{\theta f}^{1+(1 / \gamma)}\right] \leq 0
$$

where $m=$ const. $>0, n=$ const. $>0, \gamma=$ const $>1$, and then the impact angle error will converge to zero in fixed-time. The convergence rate increases as the values of $m$ and $n$ increase, and it will also increase as the value of $\gamma$ decreases. Besides, different from the finite-time convergence guidance law, the convergence time is independent of the missile's initial states.

Proof. The following continuously differential candidate function is considered:

$$
W_{2}=e_{\theta f}^{2}
$$

The derivative of (28) to time is

$$
\dot{W}_{2}=2 e_{\theta f} \dot{e}_{\theta f} \text {. }
$$

Substituting (28) and (29) into (27) yields

$$
\dot{W}_{2} \leq-m W_{2}^{1-(1 / 2 \gamma)}-n W_{2}^{1+(1 / 2 \gamma)} .
$$

According to Lemma 1, the impact angle error will converge to zero in fixed-time, and the fixed-time is given by

$$
t_{s} \leq t_{\max }=\frac{\pi \gamma}{\sqrt{m n}}
$$

where $t_{s}$ is the convergence time of the impact angle error and $t_{\max }$ is the upper bound for the settling time. It can be concluded from (31) that $t_{s}$ is independent of the initial states. Define $t_{f}$ as the final time of the engagement. To impact the target with a specific direction successfully, the error dynamics of impact angle error of the collision course should be achieved before $t_{f}$. Unlike the finite-time convergence guidance law, the convergence time $t_{s}$ is independent of the initial states, which can be adjusted by control parameters. Hence, $t_{s}<t_{f}$ can be guaranteed through proper selection of the control parameters. Figure 3 shows the convergence process of the impact angle error. It is revealed that the impact angle error converges to zero at $t_{s}$ and remains there till the end of the engagement.

Substituting (25) into (27) yields

$$
e_{\theta f}\left[\frac{u}{V}+\frac{m}{2} e_{\theta f}^{1-(1 / \gamma)}+\frac{n}{2} e_{\theta f}^{1+(1 / \gamma)}\right] \leq 0 .
$$




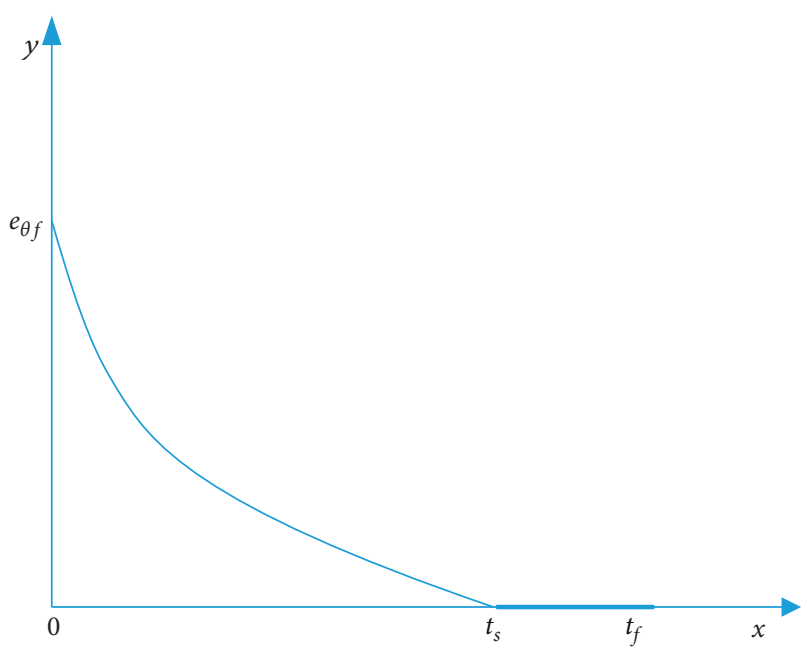

Figure 3: Impact angle error variation.

The bias term of the guidance command is chosen as

$$
u=V\left(\frac{m}{2} e_{\theta f}^{1-(1 / \gamma)}+\frac{n}{2} e_{\theta f}^{1+(1 / \gamma)}\right)+k V e_{\theta f}, \quad k>0 .
$$

Theorem 3. The bias term in equation (33) can achieve fixedtime convergence for the impact angle error.

Proof. Substituting equation (33) into equation (25), we have

$$
\dot{e}_{\theta f}=-\left(\frac{m}{2} e_{\theta f}^{1-(1 / \gamma)}+\frac{n}{2} e_{\theta f}^{1+(1 / \gamma)}\right)-e_{\theta f} .
$$

By substituting equation (34) into equation (27), we get

$$
-e_{\theta f}^{2} \leq 0
$$

According to Theorem 2, the proposed bias term in (33) can lead to fixed-time convergence for the impact angle error, and the convergence rate increases as the values of $m$ and $n$ increase or as the value of $\gamma$ decreases.

\section{Simulations}

Numerical simulation is performed to show the effectiveness of the proposed method. Three simulations are considered: In Case 1, the comparison between the proposed method and the two-stage impact angle control guidance law is considered. In Case 2, different impact angles are achieved with the same control parameters. In Case 3, three control parameters are considered to achieve the same impact angle.

The initial states for the missile and target used in this simulation are tabulated in Table 1. The simulation step for the second-order Runge-Kutta integral method applied in this study is $0.001 \mathrm{~s}$, and each simulation case will be terminated when the relative range is smaller than $0.5 \mathrm{~m}$. Besides, the maximal value for the acceleration constraint is $15 \mathrm{~g}$.
TABLE 1: Simulation parameters.

\begin{tabular}{lc}
\hline Parameter & Value \\
\hline Initial position for the missile & $(0,0) \mathrm{m}$ \\
Missile speed & $150 \mathrm{~m} / \mathrm{s}$ \\
Initial heading angle for the missile & $45^{\circ}$ \\
Initial position for the target & $(6000,0) \mathrm{m}$ \\
Maximal acceleration constraint & $15 \mathrm{~g}$ \\
\hline
\end{tabular}

4.1. Comparison Simulation. To show the effectiveness of the proposed method, one existing guidance law in Ref. [9] is considered in this comparison study. The guidance command for the comparison law is given by

$$
a^{\text {two-stage }}= \begin{cases}V \dot{\lambda}, & \text { first stage, } \\ N^{\text {two-stage }} V \dot{\lambda}, & \text { final stage. }\end{cases}
$$

The switching criterion for the comparison law is calculated from the desired impact angle, and the $N^{\text {two-stage }}=2$ for the final stage. For the proposed method, the control parameters are $m=n=0.05, \gamma=10$.

The results for this compassion simulation are shown in Figure 4. Dotted lines represent the results of the comparison guidance law, and solid lines represent the results for the proposed method. Figure 4(a) depicts the trajectories for the missile and target. Figure 4(b) shows the acceleration command. Figures 4(c) and 4(d) represent the profile of the impact angle and impact angle rate, respectively.

It can be concluded from Figure 4 that both the proposed method and the comparison law can nullify the impact angle error and impact the target with the desired impact angle successfully. However, the acceleration variation and the convergence of the heading error are significantly different, as shown in Figures 4(b) and 4(d). As for the comparison law, the guidance command will switch to another value once the heading error converges to zero. Due to the switching logic for the comparison law, the guidance command is switched to another value instantaneously; this would lead to an accumulated impact angle error. Besides, the missile cannot achieve this performance in practice with autopilot dynamics.

For the proposed guidance law, there would be no discontinuity. As a result, the proposed guidance law is more applicable and can achieve higher accuracy than the comparison law. Hence, the proposed guidance law has better performance over the comparison law.

4.2. Various Impact Angles. In this case, four different desired impact angles selected from the range $-90^{\circ}$ to $90^{\circ}$ are considered. The control parameters for the proposed guidance law are the same as those of the previous simulation.

The simulation results are shown in Figure 5. Figure 5(a) depicts the trajectories for the missile and target, and it can be concluded from the trajectories that the primary objective can be achieved by the proposed guidance law. Figure 5(b) shows impact angle variation profile, and it can be concluded each desired impact angle can be achieved, which means the 


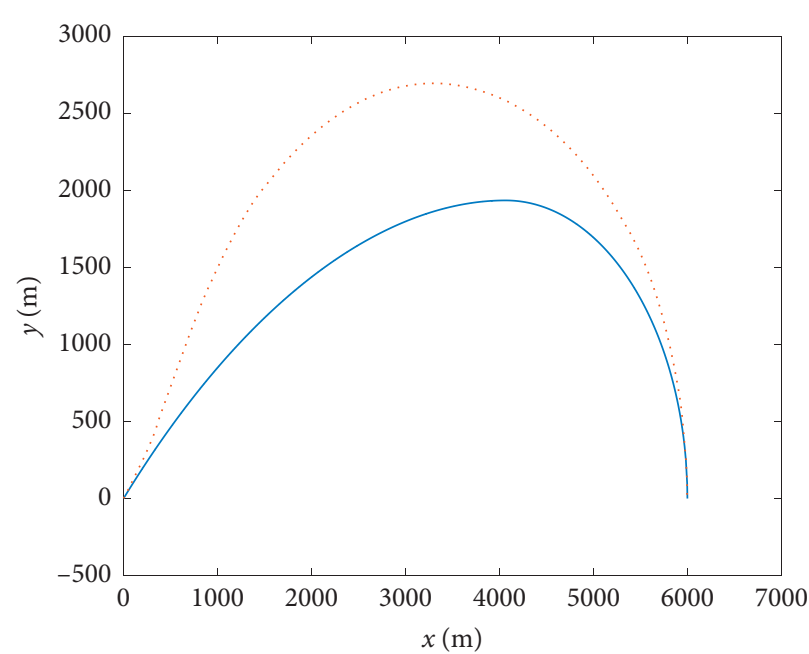

- Two-stage PN Proposed

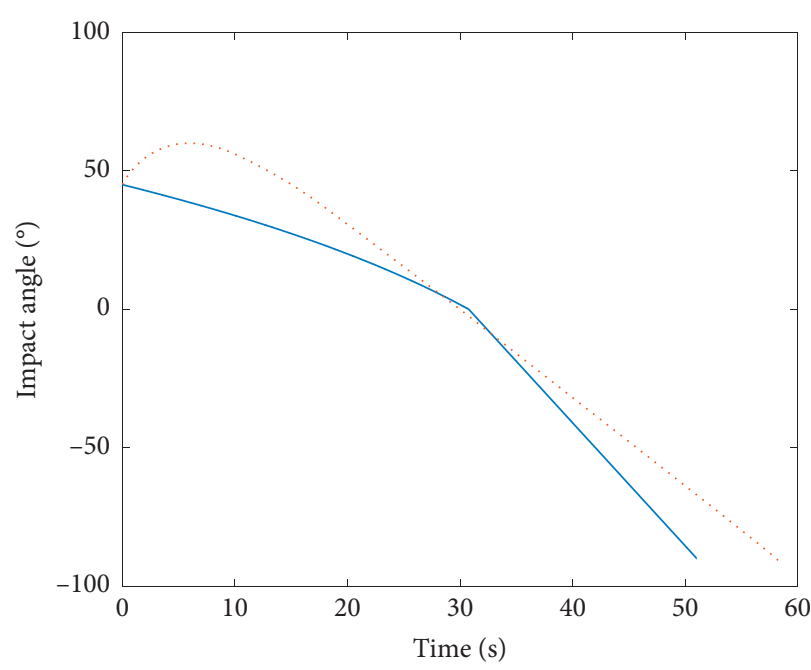

(c)

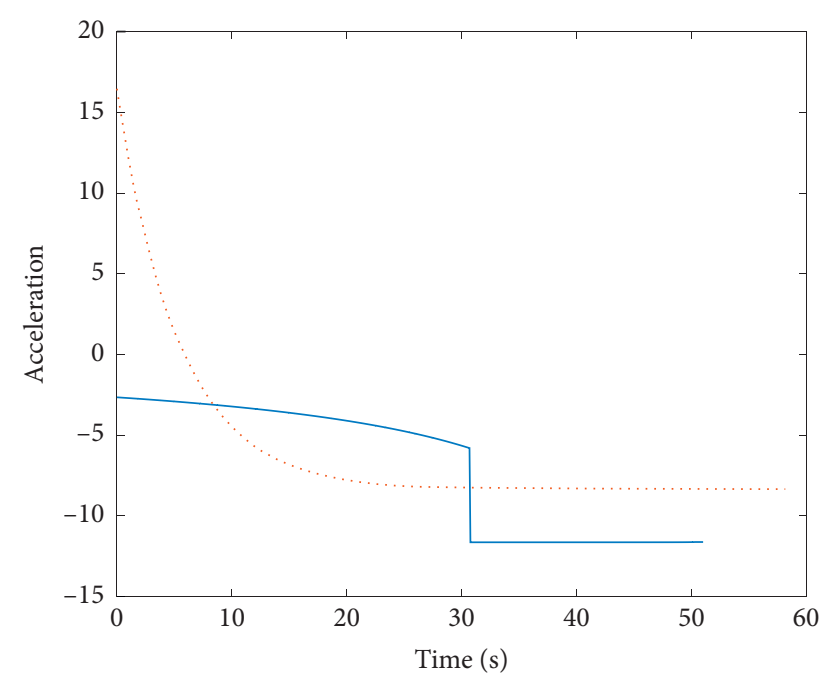

(b)

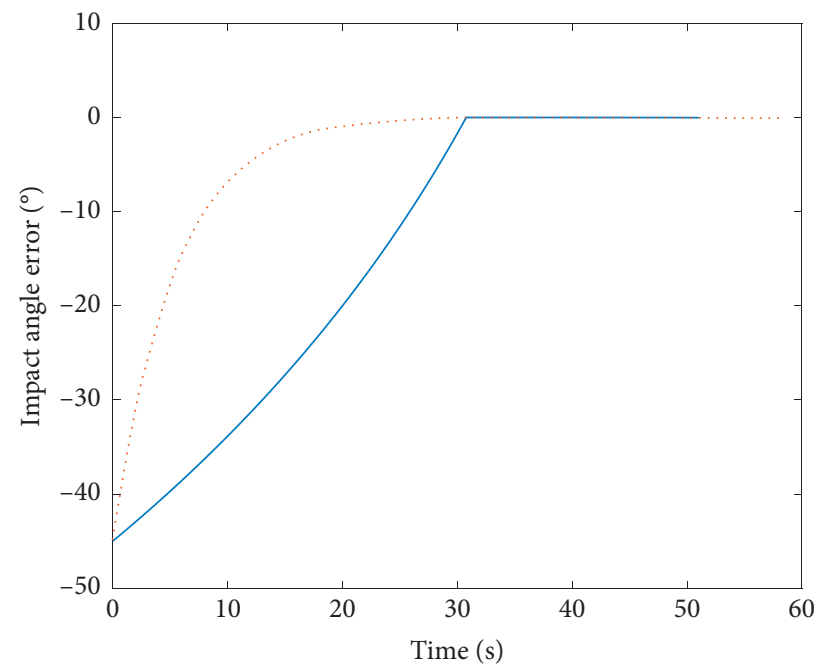

(d)

Figure 4: Comparison results. (a) Trajectories. (b) Acceleration. (c) Impact angle. (d) Impact angle error.

addition objective can also be fulfilled. Also, Figure 5(c) shows that the impact angle errors can converge to zero in fixed-time, which is in line with Theorem 3. Figure 5(d) represents the profile of the heading angle error, which converges to zero at the instant of attack, corresponding to Theorem 1. Finally, the acceleration profile is demonstrated in Figure 5(e). After the convergence of the impact angle error, the bias term in the acceleration command will be nullified.

4.3. Various Control Parameters. In this case, the performance of the proposed guidance law is studied under three different control parameters, which are $m=n=0.5, m=n=0.1, m=n=0.05, \gamma=10$.
The simulation results are shown in Figure 6. Figure 6(a) depicts the trajectories for the missile and target, and it can be concluded from the trajectories that the primary objective can be achieved by the proposed guidance law. Figure 6(b) shows the impact angle variation profile, which means the addition objective can also be fulfilled.

For all the various values of control parameters, the impact angle can converge to zero in fixed-time, as is shown in Figure 6(c), and this is in line with Theorem 3. Figure 5(d) represents the profile of the heading angle error, which converges to zero at the instant of attack, this corresponds to Theorem 1. Finally, the acceleration profile is demonstrated in Figure 5(e). After the convergence of the impact angle error, the bias term in the acceleration command will be nullified. It is obvious that the impact angle error converges 


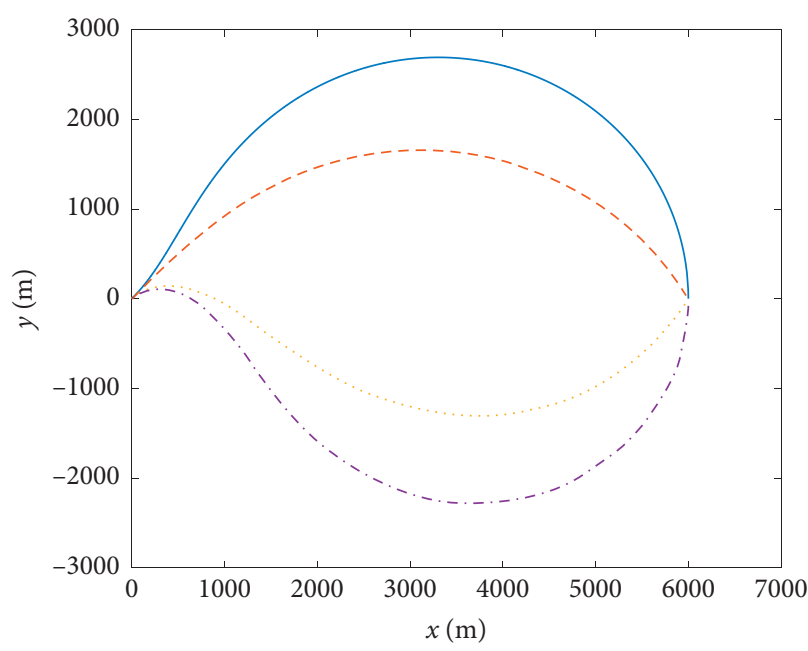
$-\Theta_{\mathrm{f}}=-90^{\circ}$
- - $\Theta_{\mathrm{f}}=-60^{\circ}$
(a) $\quad-\quad \Theta_{\mathrm{f}}=90^{\circ}$

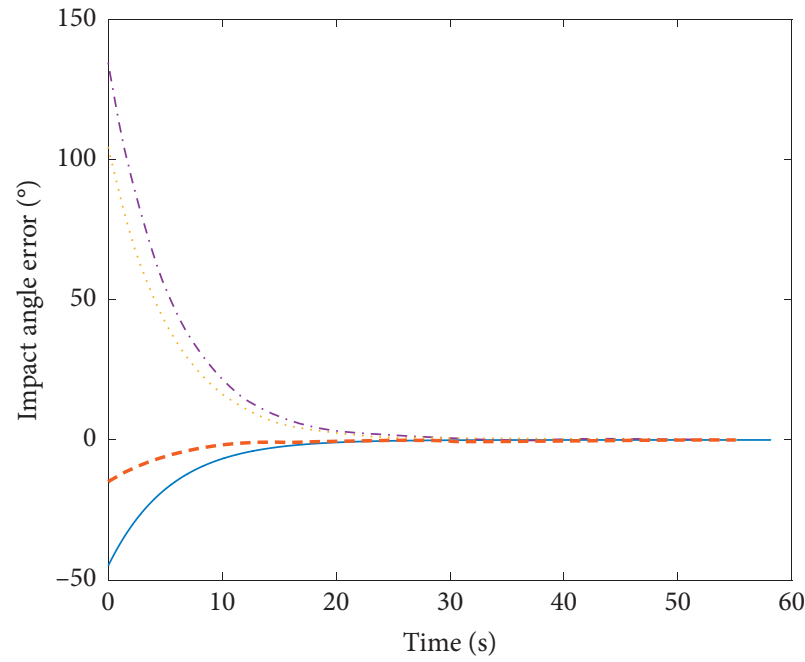

(c)

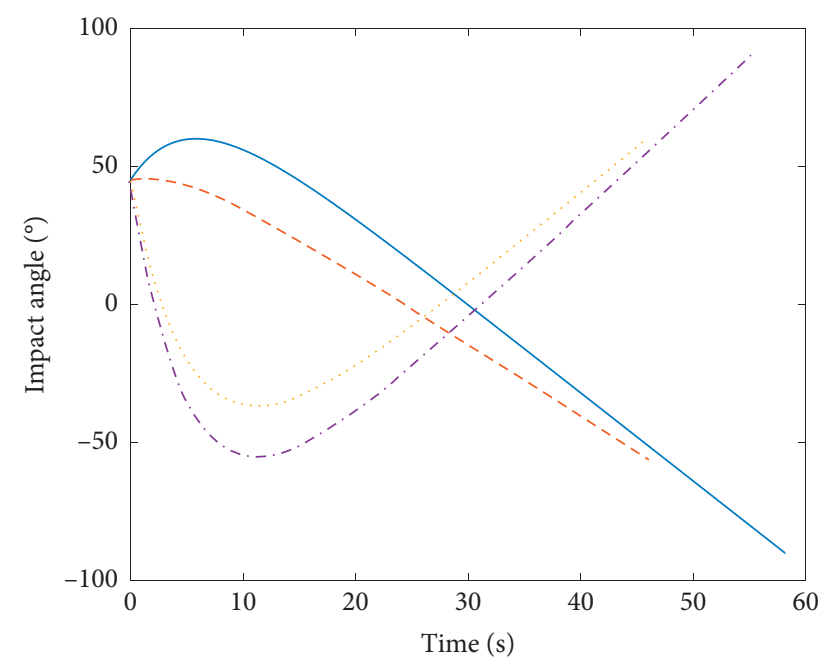

(b)

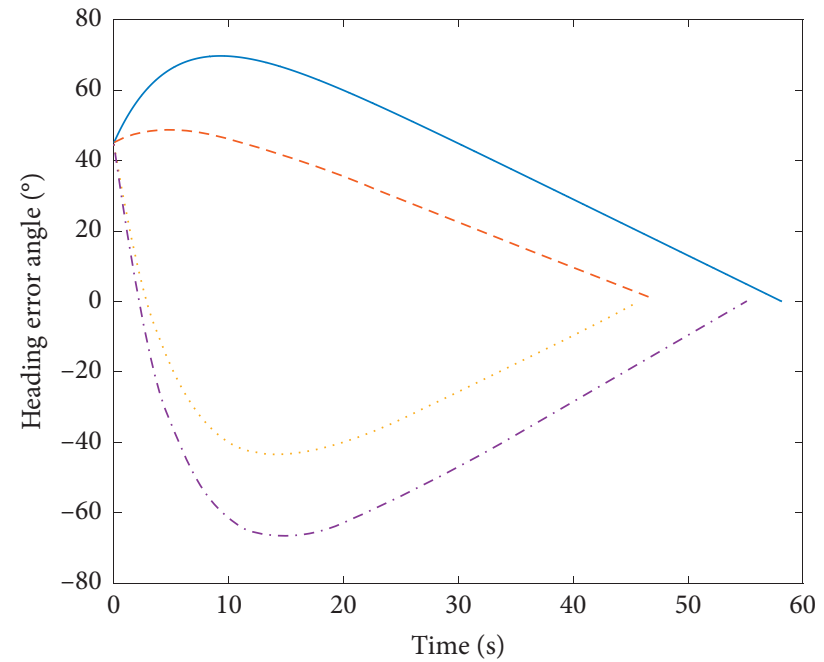

(d)

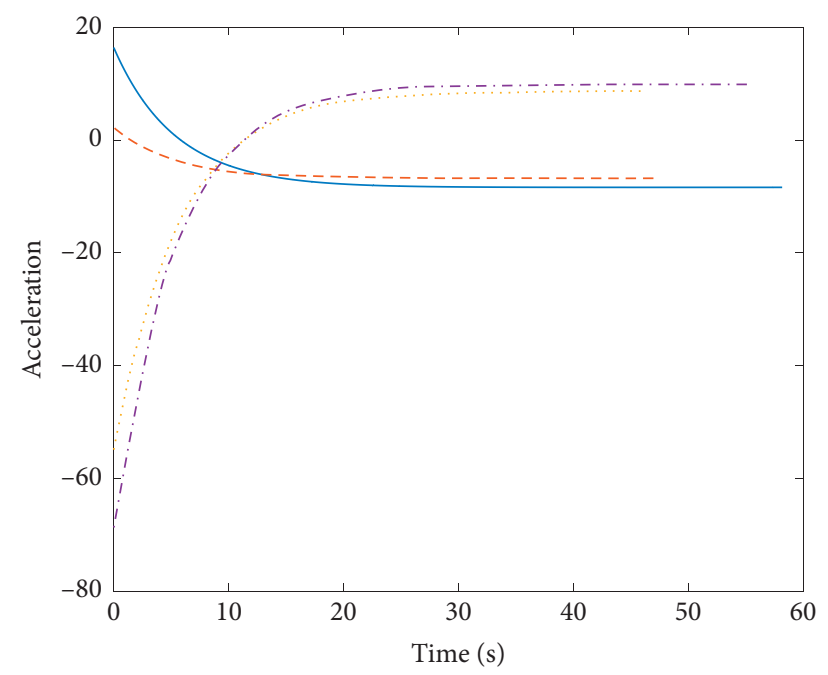

(e)

FIGURE 5: Various impact angles under the same control parameters. (a) Trajectories. (b) Impact angle. (c) Impact angle error. (d) Heading error angle. (e) Acceleration. 

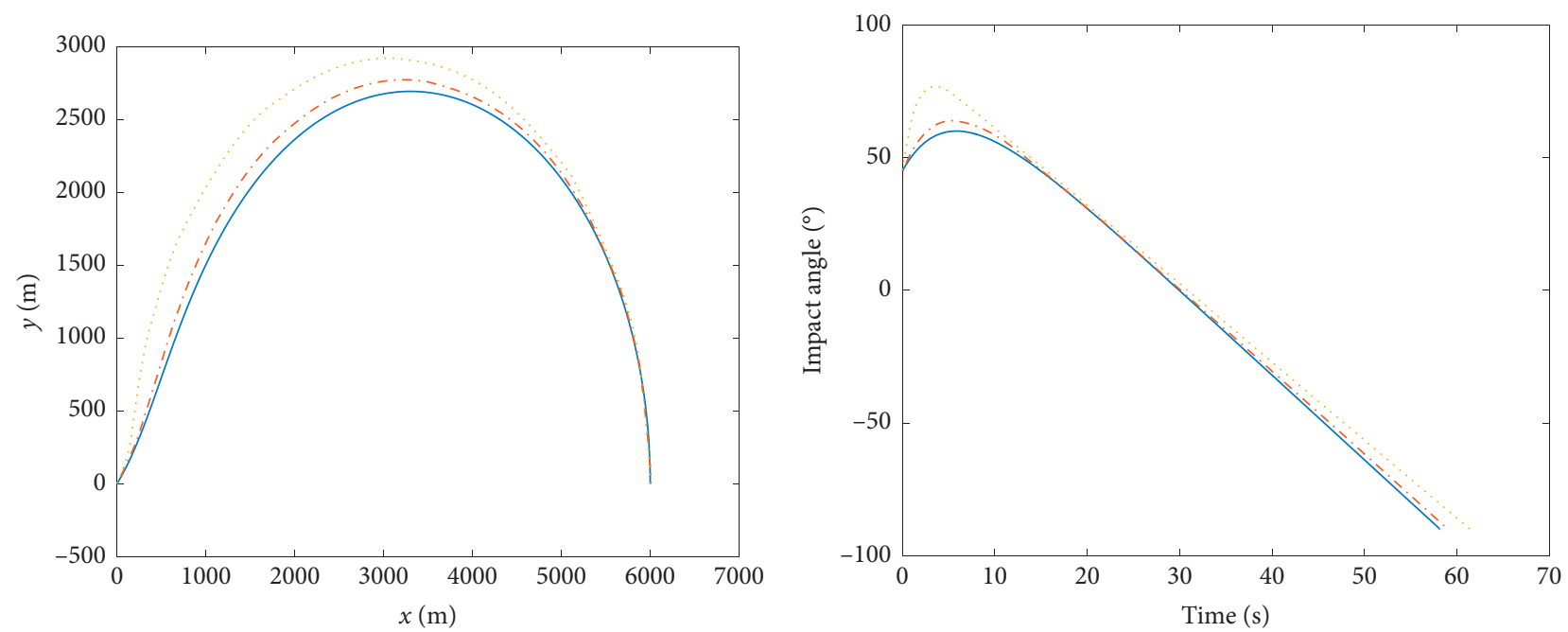

$$
\begin{aligned}
-m & =0.05, n=0.05 \\
\cdots-m & =0.1, n=0.1 \\
\cdots \cdots \quad m & =0.5, n=0.5 \quad \text { (a) }
\end{aligned}
$$

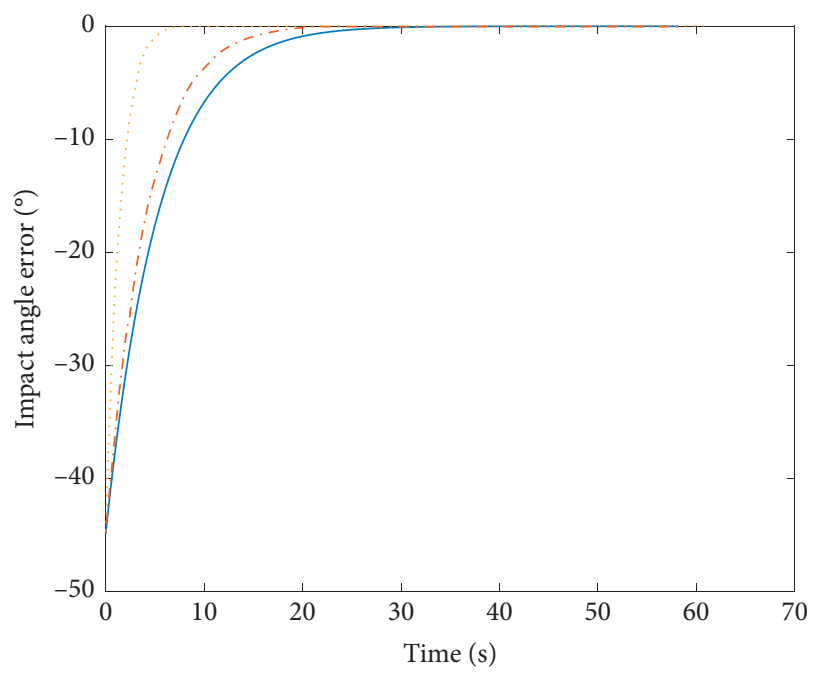

(c)

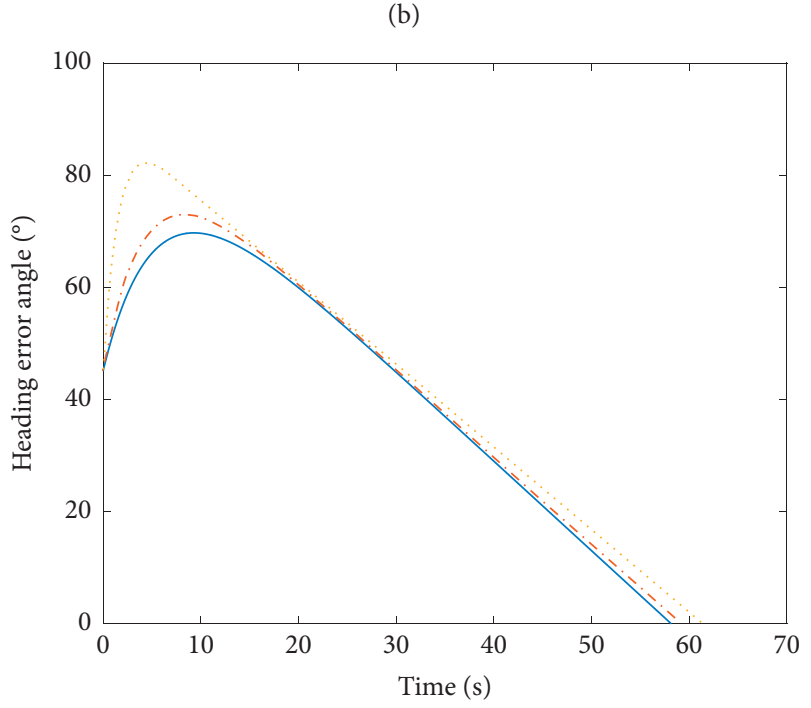

(d)

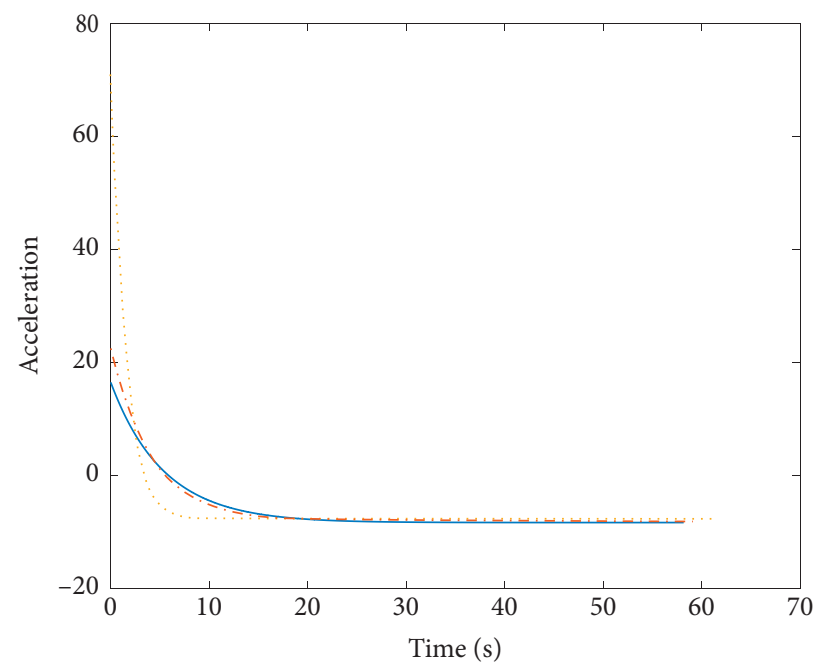

(e)

FIgURE 6: Various control parameters with the same impact angle. (a) Trajectories. (b) Impact angle. (c) Impact angle error. (d) Heading error angle. (e) Acceleration. 
more quickly with larger control parameters, and this is also in line with Theorem 1.

\section{Conclusion}

Considering the impact course can be achieved with the heading error goes to zero, the heading error shaping is applied to achieve the primary objective of the guidance law. Then, the fixed-time stability theory is utilized to ensure the convergence of the impact angle in fixed-time. The convergence rate is merely related to control parameters, a suitable selection of which can ensure the convergence before the interception. Simulations are carried out to illustrate the properties of the proposed guidance law. In our future related work, more complex scenarios such as 3-D engagement against moving targets and more constraints to improve the missile performance should also be concerned.

\section{Data Availability}

The data used to support the findings of this study are available from the corresponding author upon request.

\section{Conflicts of Interest}

The authors declare that they have no conflicts of interest.

\section{Acknowledgments}

This study was co-supported in part by the National Natural Science Foundation of China (Nos. 61903146, 61873319, and 61803162).

\section{References}

[1] C. Wei, Y. Han, N. Cui, and H. Xu, "Fifth-degree cubature kalman filter estimation of seeker line-of-sight rate using augmented-dimensional model," Journal of Guidance, Control, and Dynamics, vol. 40, no. 9, pp. 2355-2362, 2017.

[2] J. Zhou and J. Yang, "Guidance law design for impact time attack against moving targets," IEEE Transactions on Aerospace and Electronic Systems, vol. 54, no. 5, pp. 2580-2589, 2018.

[3] Z. Wu, Z. Guan, C. Yang, and J. Li, "Terminal guidance law for UAV based on receding horizon control strategy," Complexity, vol. 2017, Article ID 2750172, 19 pages, 2017.

[4] S. Mao, L. Liu, and Y. Wang., "Integrated estimation/guidance law against exoatmospheric maneuvering targets," Complexity, vol. 2018, Article ID 7470823, 19 pages, 2018.

[5] S. Gao, Z. Peng, D. Wang, and L. Liu, "Extended-state-observer-based collision-free guidance law for target tracking of autonomous surface vehicles with unknown target dynamics," Complexity, vol. 2018, Article ID 4154670, 10 pages, 2018.

[6] M. Kim and K. Grider, "Terminal guidance for impact attitude angle constrained flight trajectories," IEEE Transactions on Aerospace and Electronic Systems, vol. AES-9, no. 6, pp. 852-859, 1973.

[7] P. Lu, D. B. Doman, and J. D. Schierman, "Adaptive terminal guidance for hypervelocity impact in specified direction," Journal of Guidance, Control, and Dynamics, vol. 29, no. 2, pp. 269-278, 2006.
[8] A. Ratnoo and D. Ghose, "Impact angle constrained interception of stationary targets," Journal of Guidance Control \& Dynamics, vol. 31, no. 6, pp. 1816-1821, 2008.

[9] A. Ratnoo, "Analysis of two-stage proportional navigation with heading constraints," Journal of Guidance, Control, and Dynamics, vol. 39, no. 1, pp. 156-164, 2016.

[10] C.-K. Ryoo, H. Cho, and M.-J. Tahk, "Optimal guidance laws with terminal impact angle constraint," Journal of Guidance, Control, and Dynamics, vol. 28, no. 4, pp. 724-732, 2005.

[11] C. K. Ryoo, H. Cho, and M. J. Tahk, "Time-to-go weighted optimal guidance with impact angle constraints," IEEE Transactions on Control Systems Technology, vol. 14, no. 3, pp. 483-492, 2006.

[12] R. Tsalik and T. Shima, "Optimal guidance around circular trajectories for impact-angle interception," Journal of Guidance Control and Dynamics, vol. 39, no. 6, pp. 1-14, 2016.

[13] M. Kim and Y. Kim, "Lyapunov-based pursuit guidance law with impact angle constraint," IFAC Proceedings Volumes, vol. 47, no. 3, pp. 2509-2514, 2014.

[14] C. Zhongtao, L. Lei, and W. Yongji, "Lyapunov-based switched-gain impact angle control guidance," Chinese Journal of Aeronautics, vol. 31, no. 4, pp. 765-775, 2018.

[15] Y. Zhang, M. Sun, and Z. Chen, "Finite-time convergent guidance law with impact angle constraint based on slidingmode control," Nonlinear Dynamics, vol. 70, no. 1, pp. 619$625,2012$.

[16] Z. Hou, L. Liu, Y. Wang, J. Huang, and H. Fan, “Terminal impact angle constraint guidance with dual sliding surfaces and model-free target acceleration estimator," IEEE Transactions on Control Systems Technology, vol. 25, no. 1, pp. 85-100, 2017.

[17] S. He, D. Lin, and J. Wang, "Continuous second-order sliding mode based impact angle guidance law," Aerospace Science and Technology, vol. 41, pp. 199-208, 2015.

[18] B.-M. Min, M.-J. Tahk, H.-C. D. Shim, and H.-C. Bang, "Guidance law for vision-based automatic landing of UAV," International Journal of Aeronautical and Space Sciences, vol. 8, no. 1, pp. 46-53, 2007.

[19] C.-H. Lee, T.-H. Kim, M.-J. Tahk, and I.-H. Whang, "Polynomial guidance laws considering terminal impact angle and acceleration constraints," IEEE Transactions on Aerospace and Electronic Systems, vol. 49, no. 1, pp. 74-92, 2013.

[20] T.-H. Kim, C.-H. Lee, I.-S. Jeon, and M.-J. Tahk, "Augmented polynomial guidance with impact time and angle constraints," IEEE Transactions on Aerospace and Electronic Systems, vol. 49, no. 4, pp. 2806-2817, 2013.

[21] Z. Cheng, B. Wang, L. Liu, and Y. Wang, "Adaptive polynomial guidance with impact angle constraint under varying velocity," IEEE Access, vol. 7, pp. 104210-104217, 2019.

[22] A. Polyakov, "Nonlinear feedback design for fixed-time stabilization of linear control systems," IEEE Transactions on Automatic Control, vol. 57, no. 8, pp. 2106-2110, 2012. 